\title{
IncRNA LIFR-AS1 suppresses invasion and metastasis of non-small cell lung cancer via the miR-942-5p/ZNF471 axis
}

\author{
Qun Wang ${ }^{1,2,3+}$, Jing Wu ${ }^{2+}$, Hui Huang ${ }^{2+}$, Yan Jiang ${ }^{4}$, Ying Huang ${ }^{2}$, Hongyan Fang ${ }^{2}$, Gang Zheng ${ }^{5}$, \\ Xiaochun Zhou ${ }^{2}$, Yujuan $\mathrm{Wu}^{2}$, Changjiang Lei ${ }^{5}$ and Desheng $\mathrm{Hu}^{1 *}$ (D)
}

\begin{abstract}
Background: MicroRNA 942-5p (miR-942-5p) has been reported to promote migration and invasion in non-small cell lung cancer (NSCLC), but the underlying mechanism is not completely understood. The interplay between long noncoding RNAs (IncRNAs) and miRNAs plays a crucial role in tumor progression.

Methods: In the present study, we performed bioinformatic and biochemical analyses to identify miR-942-5p-interacting IncRNAs. The function and clinical significance of the candidate IncRNA(s) in NSCLC were determined.

Results: We identified LIFR-AS1 as a pivotal miR-942-5p-interacting IncRNA. Overexpression of miR-942-5p caused a reduction of LIFR-AS1 in NSCLC cells. LIFR-AS1 showed the ability to sponge miR-942-5p, leading to derepression of ZNF471. Functionally, LIFR-AS1 overexpression inhibited NSCLC cell migration and invasion, whereas LIFR-AS1 silencing yielded an opposite effect. In vivo studies confirmed that LIFR-AS1 overexpression suppressed lung metastasis of NSCLC cells. Rescue experiments demonstrated that enforced expression of miR-942-5p or depletion of ZNF471 restored the migration and invasion capacity of LIFR-AS1-overexpressing cells. Moreover, overexpression of ZNF471 restrained NSCLC cell invasion. Clinically, LIFR-AS1 downregulation was significantly correlated with TNM stage, lymph node metastasis, and reduced overall survival in NSCLC patients.
\end{abstract}

Conclusions: we provide first evidence for the involvement of the LIFR-AS1/miR-942-5p/ZNF471 axis in NSCLC invasion and metastasis. LIFR-AS1 may represent a novel target for the treatment of NSCLC.

Keywords: Lung cancer, IncRNA, Metastasis, miR-942-5p, Target gene

\section{Background}

Non-small cell lung cancer (NSCLC), accounting for more than $85 \%$ of all lung cancers, is one of the leading causes of cancer-related mortality [1]. The 5-year overall survival rate for patients with NSCLC is approximately $15 \%$ [2]. The poor prognosis of NSCLC is mainly

\footnotetext{
*Correspondence: 13907174495@163.com

${ }^{\dagger}$ Qun Wang, Jing Wu and Hui Huang contributed equally to this work ${ }^{1}$ Department of Radiotherapy, Hubei Cancer Hospital, Tongji Medical College, Huazhong University of Science and Technology, 116 Zhuodaoquan South Road, Wuhan 430070, China

Full list of author information is available at the end of the article
}

attributed to cancer cell invasion and metastasis [3]. Hence, exploring the molecular mechanism underlying NSCLC metastasis is of significance in treating this malignancy.

Zinc-finger protein 471 (ZNF471), belonging to the large family of zinc-finger proteins, is frequently downregulated in tumors due to promoter hypermethylation $[4,5]$. Bhat et al. [4] demonstrated that downregulation of ZNF471 is significantly associated with reduced survival in patients with head and neck squamous cell carcinoma. ZNF471 has exhibited tumor-suppressive activities in

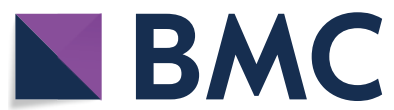

(c) The Author(s) 2020. This article is licensed under a Creative Commons Attribution 4.0 International License, which permits use, sharing, adaptation, distribution and reproduction in any medium or format, as long as you give appropriate credit to the original author(s) and the source, provide a link to the Creative Commons licence, and indicate if changes were made. The images or other third party material in this article are included in the article's Creative Commons licence, unless indicated otherwise in a credit line to the material. If material is not included in the article's Creative Commons licence and your intended use is not permitted by statutory regulation or exceeds the permitted use, you will need to obtain permission directly from the copyright holder. To view a copy of this licence, visit http://creativeco mmons.org/licenses/by/4.0/. The Creative Commons Public Domain Dedication waiver (http://creativecommons.org/publicdomain/ zero/1.0/) applies to the data made available in this article, unless otherwise stated in a credit line to the data. 
gastric cancer [5] and esophageal cancer [6]. These studies indicate ZNF471 as a tumor suppressor.

Long non-coding RNAs (lncRNAs) represent a large class of non-coding RNAs of longer than 200 nucleotides [7]. In recent years, lncRNAs have attracted attention as important regulators of biological processes such as cell proliferation, differentiation, inflammation, infection, and tumor progression [7-9]. Most interestingly, lncRNAs can coordinate NSCLC growth and metastasis $[8,9]$. For example, the IncRNA GIAT4RA has been reported to inhibit NSCLC cell growth, colony formation, migration, and invasion [8]. LIFR-AS1 is located on human chromosome $5 \mathrm{p} 13.1$. A previous study reported that LIFR-AS1 inhibits the proliferation and survival of colorectal cancer cells [10]. Another study demonstrated that LIFR-AS1 has the ability to control breast cancer cell proliferation and migration [11]. However, the role of LIFR-AS1 in NSCLC remains unclear.

It has been suggested that lncRNAs can exert their biological effects by sponging microRNAs (miRNAs) to regulate target gene expression [9]. miRNAs are small, endogenous non-coding RNAs that are capable of repressing gene expression by binding to the $3^{\prime}$-untranslated region (3'-UTR) of target mRNAs [10]. Compelling evidence indicates that lncRNAs can work as competitive endogenous RNA (ceRNA) for miRNAs [9, 11, 12]. For example, the lncRNA MALAT1 antagonizes the activity of miR-199a to promote ZHX1 expression, leading to increased glioblastoma cell proliferation and survival [12].

miR-942-5p plays an oncogenic role in multiple cancer types including esophageal squamous cell cancer [13], colorectal cancer [14], hepatocellular carcinoma [15], and breast cancer [16]. Yang et al. [17] confirm the tumor-promoting activity of miR-942-5p in NSCLC. The lncRNAs ADAMTS9-AS2 [18] and LINC00675 [14] have been reported to sponge miR-942-5p in mesenchymal stem cells and colorectal cancer cells, respectively. However, overexpression of either of the 2 lncRNAs did not affect the expression of miR-942-5p in NSCLC cells (data not shown). Thereby, in the present study, we aim to identify novel miR-942-5p-interacting lncRNAs and explore their function in NSCLC.

\section{Methods and materials Cell culture}

Human NSCLC cell lines A549, H1299, PC-9, and H1975 and human bronchial epithelial cell line BEAS-2B were purchased from the Cell Bank of Shanghai Institute of Cell Biology, Chinese Academy of Sciences (Shanghai, China). NSCLC cells were cultured in Dulbecco's modified Eagle Medium (DMEM, Invitrogen, Carlsbad, CA, USA) with $10 \%$ fetal bovine serum (FBS; Invitrogen).
BEAS-2B cells were grown in growth factor-supplemented medium (BEGM; Lonza, Walkersville, MD, USA). All the cell lines were maintained in a humidified atmosphere of $5 \% \mathrm{CO}_{2}$ at $37^{\circ} \mathrm{C}$.

\section{Oligonucleotides, plasmids, and transfections}

MiR-942-5p mimic and negative control mimic were purchased from Sigma-Aldrich (St. Louis, MO, USA). The sequences of LIFR-AS1 (NR_103553.1) and ZNF471 (NM_020813.4) were inserted to pcDNA3.1(+) expression vector. Short hairpin RNAs (shRNAs) targeting LIFR-AS1 and ZNF471 were synthesized by Shanghai Sangon Company (Shanghai, China) and cloned to the pLKO.1 vector. The wild-type LIFR-AS1 and ZNF471 $3^{\prime}$-UTR luciferase reporters were constructed in the pmirGLO plasmid (Promega, Madison, WI, USA). The QuikChange Site-Directed Mutagenesis Kit (Stratagene, La Jolla, CA, USA) was utilized to generate a mutated LIFR-AS1 or ZNF471 3'-UTR with disruption of the putative miR-942-5p binding site. All the constructs were validated by sequencing. They were transfected to NSCLC cell lines using Lipofectamine 3000 transfection reagent (Invitrogen) according to the manufacturer' $\mathrm{s}$ instruction.

\section{Quantitative real-time PCR (qRT-PCR)}

Total RNA was extracted from tissues and cells using TRIzol Reagent (Invitrogen). Reverse transcription was carried out using the QuantiTect Reverse Transcription Kit (Qiagen, Hilden, Germany). The resultant cDNA was amplified using a SYBR Green PCR Kit (Qiagen). The PCR primers used are as follows: LIFR-AS1 forward $5^{\prime}$-GCAAATACTGTGTATTAGTCC-3' ${ }^{\prime}$ and LIFRAS1 reverse 5'-CCGCTTCCTTGTGAAGAAGGT-3'; ZNF471 forward 5'-CACAGCTGGCTACTCATCAGA$3^{\prime}$ and ZNF471 reverse 5'-GCTGAAGGCTTTCCCGCA TTC-3'; RND3 forward 5'-CTATGACCAGGGGGC AAATA- $3^{\prime}$ and RND3 reverse $5^{\prime}$-TCTTCGCTTTGT CCTTTCGT-3'; CCBE1 forward 5'-AGGCGACACTCC ACAGT- $3^{\prime}$ and CCBE1 reverse 5'-GATTAGTGGTCG CTATATT-3'; KDM5A forward 5'-GATGACAGCATG GAAGAGAAAC- $3^{\prime}$ and KDM5A reverse 5'-GCCAGT TTATTCAGCTCCTTTG-3'; GAPDH forward 5'-ACC ACAGTCCATGCCATCAC- $3^{\prime}$ and GAPDH reverse $5^{\prime}$-TCCACCACCCTGTTGCTGTA-3'. For detection of miR-942-5p, cDNA was synthesized by the TaqMan MicroRNA Reverse Transcription Kit (Applied Biosystems, Foster City, CA, USA)according to the manufacturer's instruction. RNA expression was quantified using the mirVana qRT-PCR microRNA Detection Kit (Applied Biosystems). U6 was used as the normalization control. 
The relative expression levels of the target genes were determined by the $2^{-\Delta \Delta \mathrm{Ct}}$ method [19].

\section{Luciferase reporter assay}

Luciferase reporter assay was performed as described previously [20]. The LIFR-AS1 and ZNF471 3 '-UTR luciferase vectors were co-transfected with miR-942-5p mimic or negative control mimic to NSCLC cells using Lipofectamine 3000 . The cells were collected $48 \mathrm{~h}$ after transfection, and the luciferase activities were measured using the Dual-Luciferase Reporter Assay System (Promega). The relative luciferase activity was presented after normalization to the activity of Renilla luciferase.

\section{RNA immunoprecipitation (RIP) assay}

RIP assay was performed as described previously [21]. In brief, A549 and H1299 cells were lysed using RIP immunoprecipitation buffer containing protease/RNase inhibitors (Roche, Madison, WI, USA). In some settings, A549 cells were transfected with miR-942-5p mimic or control mimic together with wild-type or mutated ZNF471. The lysates were then subjected to immunoprecipitation using anti-Ago 2 monoclonal antibody or mouse IgG control (Sigma-Aldrich) conjugated to magnetic beads. After incubation at $4{ }^{\circ} \mathrm{C}$ overnight, the immunoprecipitated RNA was extracted with Trizol Reagent from the beads and quantitated by qRT-PCR analysis.

\section{Western blot analysis}

Cells were lysed using radioimmunoprecipitation assay buffer with a protease inhibitor cocktail (Roche). Protein concentrations were measured using the Pierce BCA Protein Assay Kit (Thermo Fisher Scientific, Waltham, MA, USA). Protein samples were resolved by SDS-polyacrylamide gel electrophoresis. Rabbit anti-ZNF471/ERP1 (ab204974) and anti- $\beta$-actin (ab8227) antibodies (Abcam, Cambridge, MA, USA) were used as the primary antibodies. Protein band was visualized by ECL chemiluminescent reagent (Millipore, Billerica, MA, USA).

\section{Wound-healing assay}

Cell migration was determined using wound-healing assay, as described previously [22]. In brief, transfected cells were plated into 6-well plates and grown to confluence. The cell monolayers were wounded with a pipette tip, and cell debris was rinsed off. $10 \mu \mathrm{g} / \mathrm{ml}$ mitomycin C (Sigma-Aldrich) was added to inhibit cell proliferation. Representative images were captured at 0 and $48 \mathrm{~h}$ after injury. The percentage of wound healing was quantified. All experiments were repeated three times.

\section{Transwell invasion assay}

Transwell invasion assay was conducted as described previously [23]. In brief, $5 \times 10^{4}$ cells in serum-free medium were plated into the upper chamber precoated with Matrigel (BD Biosciences, San Jose, CA, USA). The lower chambers were added with DMEM supplemented with $10 \%$ FBS. After incubation for $48 \mathrm{~h}$ at $37{ }^{\circ} \mathrm{C}$, the cells that had invaded were fixed with $4 \%$ paraformaldehyde and stained with $0.5 \%$ crystal violet. Cells were counted in 5 representative fields per insert under a microscope.

\section{Animal experiments}

For in vivo lung metastasis assay, luciferase-labeled NSCLC cells were intravenously injected into nude mice as described previously [24]. In brief, LIFR-AS1overexpressing or empty vector-transfected PC-9 cells were stably transfected with a luciferase expression construct. The transfected cells $\left(2 \times 10^{6}\right)$ were injected through the tail vein. Luciferase activity was monitored using the IVIS Spectrum imaging system (Perkin-Elmer Life Sciences, Waltham, MA, USA). Bioluminescence images were acquired 6 weeks after cell injection. The mice were then euthanized, and the lung tissues were harvested and subjected to hematoxylin and eosin (H\&E) staining.

\section{Tissue specimens}

We collected 73 pairs of NSCLC and corresponding normal lung tissues from patients with NSCLC who

\begin{tabular}{|c|c|c|c|c|}
\hline \multirow[t]{2}{*}{ Variable } & \multirow[t]{2}{*}{$\mathbf{n}$} & \multicolumn{2}{|l|}{ LIFR-AS1 } & \multirow[t]{2}{*}{$P$} \\
\hline & & $\begin{array}{l}\text { Low } \\
\text { expression } \\
(n=43)\end{array}$ & $\begin{array}{l}\text { High } \\
\text { expression } \\
(n=30)\end{array}$ & \\
\hline Age & & & & 0.8340 \\
\hline$<65$ & 33 & 19 & 14 & \\
\hline$\geq 65$ & 40 & 24 & 16 & \\
\hline Sex & & & & 0.4716 \\
\hline Male & 52 & 32 & 10 & \\
\hline Female & 21 & 11 & 10 & \\
\hline TNM stage & & & & 0.0321 \\
\hline$|-| \mid$ & 48 & 24 & 24 & \\
\hline III-IV & 25 & 19 & 6 & \\
\hline Lymph node metastasis & & & & 0.0047 \\
\hline Negative & 47 & 22 & 25 & \\
\hline Positive & 26 & 21 & 5 & \\
\hline
\end{tabular}


underwent surgical resection between July 2017 and July 2018. None received any anticancer treatment before surgery. Clinicopathological information is shown in Table 1.

\section{Statistical analysis}

All values are reported as mean \pm standard deviation and analyzed by the Student's $t$ test or one-way analysis of variance followed by the post hoc Tukey's test. The correlation of LIFR-AS1 expression with clinicopathological characteristics was calculated with the Chi square test. $P<0.05$ was considered statistically significant.

\section{Results}

LIFR-AS1 is reduced by miR-942-5p in NSCLC cells

To identify miR-942-5p-interacting lncRNAs in NSCLC, we examined the effect of miR-942-5p overexpression on the expression of 21 novel candidates (Additional file 1: Table S1) in A549 cells. These candidate lncRNAs were predicted using the Encyclopedia of RNA Interactomes (ENCORI) program (http://starbase.sysu.edu.cn/index .php). As shown in Fig. 1a, only LIFR-AS1 was downregulated by ectopic expression of miR-942-5p. In contrast, the other 20 lncRNAs tested remained unchanged (data not shown). We also validated the miR-942-5p-induced downregulation of LIFR-AS1 in another NSCLC cell line, H1299 (Fig. 1a). These findings indicate a negative regulation of LIFR-AS1 by miR-942-5p. However, LIFRAS1 overexpression did not alter the expression of miR942-5p (Additional file 1: Figure S1).

Bioinformatic analysis predicted a putative binding site for miR-942-5p in LIFR-AS1 (Fig. 1b). To validate the direct interaction between LIFR-AS1 and miR-942-5p, we transfected the LIFR-AS1 luciferase reporters together with miR-942-5p mimic into A549 and H1299 cells. The results showed that miR-942-5p overexpression repressed the luciferase reporter harboring the wild-type LIFR-AS1 fragment, while had no effect on the mutated LIFR-AS1 luciferase reporter (Fig. 1c, d). Altogether, these data confirm that miR-942-5p can target LIFR-AS1 in NSCLC cells.

\section{LIFR-AS1 sponges miR-942-5p to upregulate ZNF471}

It is well known that miRNAs regulate gene expression through the miRNA-induced silencing complex (miRISC), which comprises the effector molecules, argonaute (Ago) proteins [25]. To confirm the binding

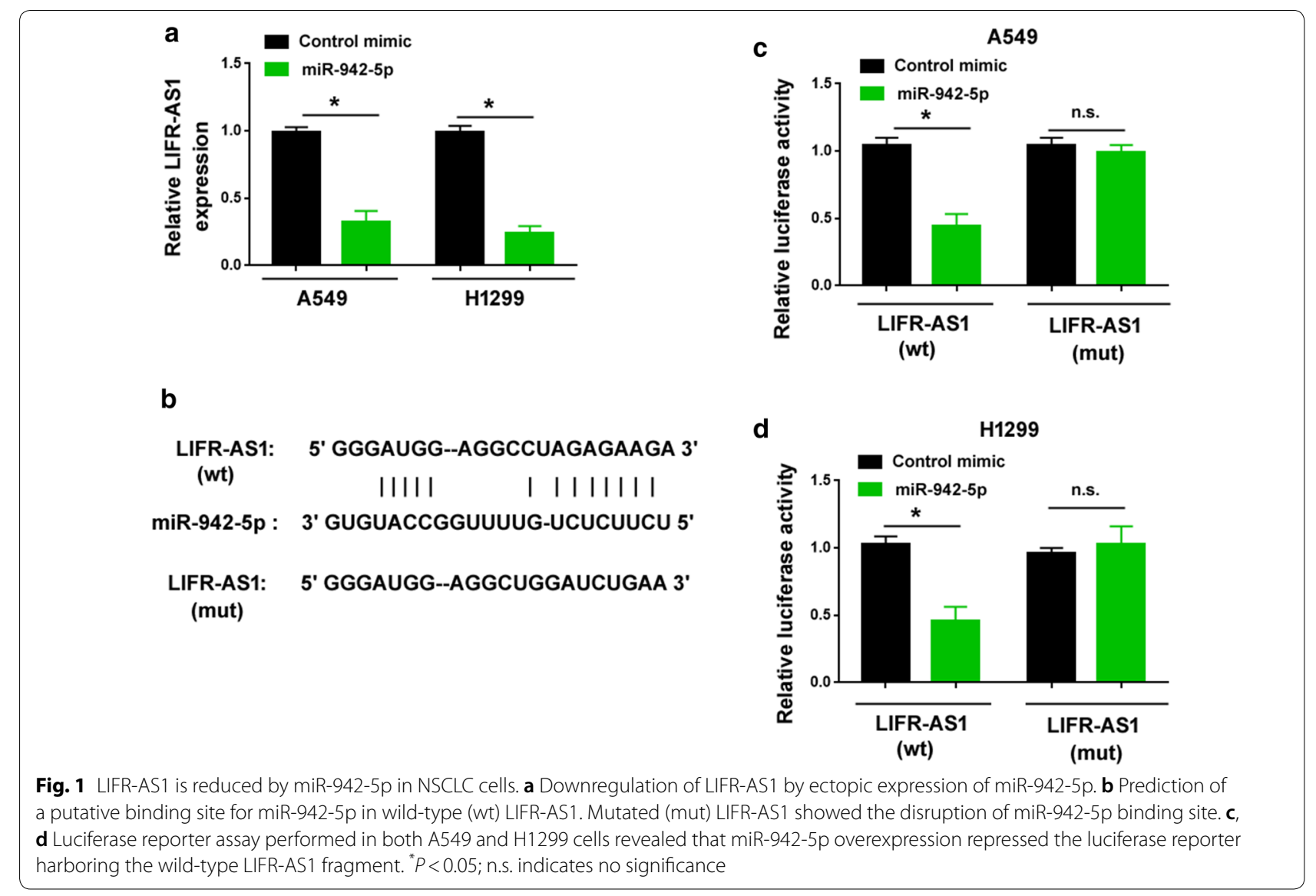



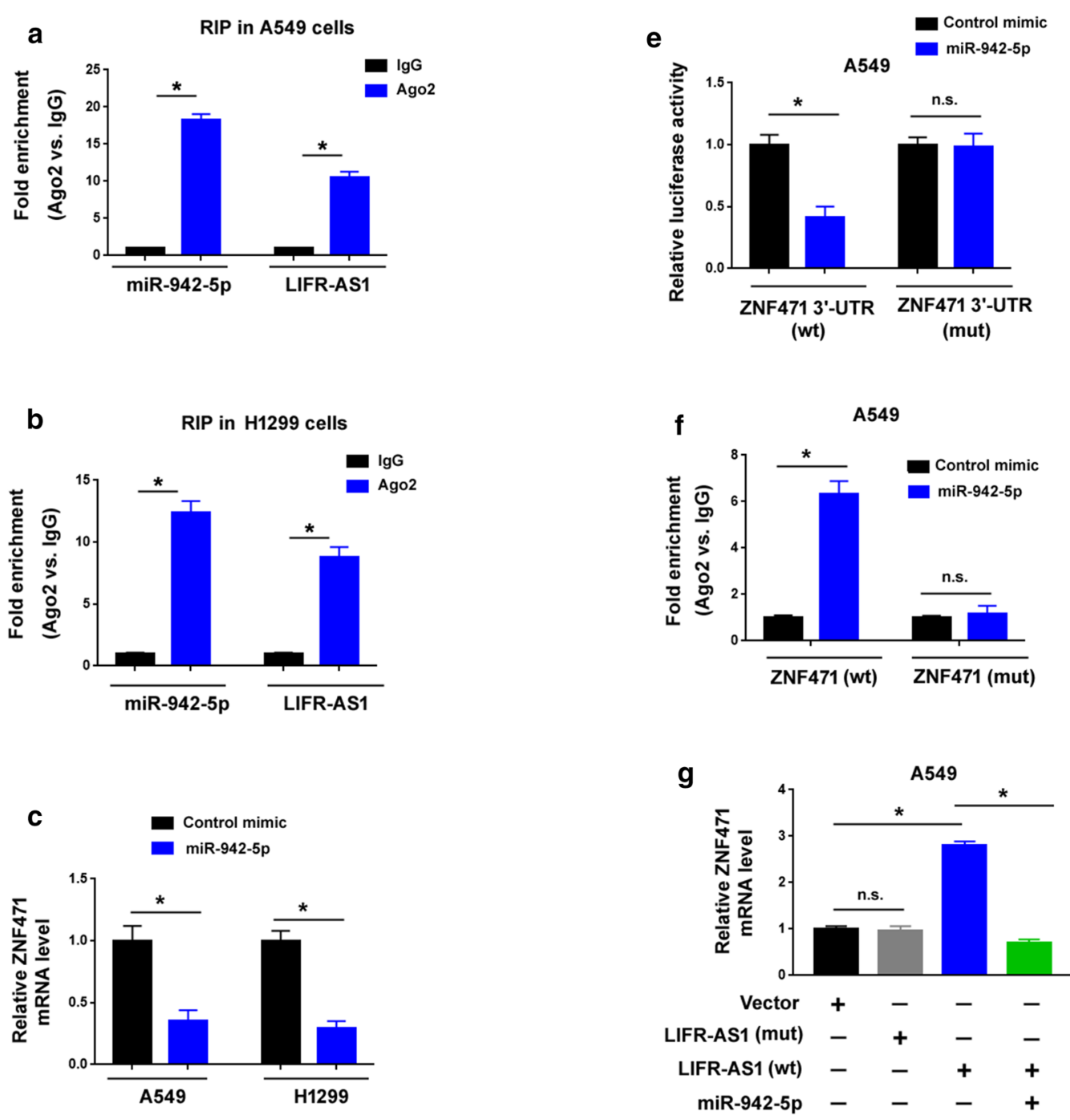
d
ZNF471 3'-UTR 5 ' ...UAGAAUAACCAGUUUAGAGAAGA...
(wt)
| | | | | || | |
miR-942-5p 3' GUGUACCGgUUUUGUCUCUUCU
ZNF471 3'-UTR 5' ...UAGAAUAAGUCGUUCAUACCUAA... (mut)

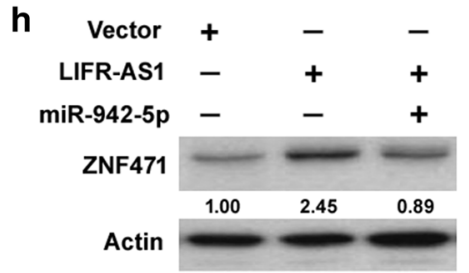

Fig. 2 LIFR-AS1 sponges miR-942-5p to upregulate ZNF471. a, b RIP assays performed with anti-Ago2 antibody showed that miR-942-5p and LIFR-AS1 were detected in Ago2 immunoprecipitates from both A549 and H1299 cells. c Overexpression of miR-942-5p decreased the mRNA level of ZNF471 in both A549 and H1299 cells. d Bioinformatic analysis predicted that the wild-type (wt) 3'-UTR of ZNF471 harbored a potential miR-942-5p binding site. A mutated (mut) ZNF471 3'-UTR was constructed by disruption of the miR-942-5p binding site. e Luciferase reporter assays showed that overexpression of miR-942-5p decreased the luciferase activity of the reporter with the wild-type but not mutated ZNF471 3'-UTR. f Ago2 RIP assays were performed in A549 cells transfected with miR-942-5p mimic together with wild-type or mutated ZNF471. Results are expressed as fold enrichment relative to control mimic. $\mathbf{g}$ Wild-type but not mutated LIFR-AS1 promoted the mRNA expression of ZNF471, which was reversed by miR-942-5p overexpression. $\mathbf{h}$ Western blot analysis of ZNF471 protein levels in A549 cells transfected with indicated constructs. ${ }^{*} P<0.05 ;$ n.s. indicates no significance 
of miR-942-5p to LIFR-AS1, we performed RIP assays in A549 and H1299 cells using anti-Ago2 antibody. Of note, miR-942-5p and LIFR-AS1 were detected in Ago2 immunoprecipitates, as determined by qRT-PCR analysis (Fig. 2a, b). This result raises the possibility that LIFR-AS1 might act as a sponge of miR-942-5p, thereby regulating target gene expression. A previous study has suggested BARX2 as a direct target of miR-942-5p [17]. However, we did not observe the regulation of BARX2 by LIFR-AS1 in NSCLC cells (Additional file 1: Figure S2). Therefore, we investigated the impact of LIFR-AS1 on other miR-942-5p target genes. Based on the TargetScan program (http://www.targetscan.org/vert_71/), we predicted a large number of target genes for miR-942-5p. Among them, we focused on the genes that were positively correlated with LIFR-AS1 based on The Cancer Genome Atlas (TCGA) data. We then selected the top 25 candidates for further validation. Intriguingly, overexpression of miR-942-5p led to a significant decline in the mRNA level of ZNF471 in both A549 and H1299 cells (Fig. 2c), but had no impact on the other 24 mRNAs including RND3, KDM5A, and CCBE1 (Additional file 1: Figure S3).

To verify the binding between ZNF471 and miR942-5p, we performed luciferase reporter assays using the luciferase reporter carrying the $3^{\prime}$-UTR of ZNF471 (Fig. 2d, e). Overexpression of miR-942-5p decreased the luciferase activity of the reporter with the wild-type ZNF471 3'-UTR, but not that with a mutated ZNF471 $3^{\prime}$-UTR (Fig. 2e). To validate the binding of miR-942-5p to ZNF471 mRNA, we performed Ago2 RIP assays in A549 cells transfected with miR-942-5p mimic or control mimic together with wild-type or mutated ZNF471. The enrichment of wild-type but not mutated ZNF471 in Ago2 immunoprecipitates was observed when miR942-5p was overexpressed in A549 cells (Fig. 2f). In addition, qRT-PCR analysis indicated that ZNF471 mRNA expression was significantly upregulated in LIFR-AS1overexpressing NSCLC cells (Fig. 2g). Such upregulation was reversed by overexpression of miR-942-5p (Fig. 2g). However, a mutant LIFR-AS1 with disrupted miR-942-5p binding did not affect the abundance of ZNF471 mRNA. LIFR-AS1-mediated induction of ZNF471 expression was also validated at the protein level (Fig. 2h). These data collectively suggest that LIFR-AS1 can competitively bind with miR-942-5p to derepress ZNF471 in NSCLC cells.

\section{LIFR-AS1 suppresses NSCLC cell invasion and metastasis}

To dissect the role of LIFR-AS1 in NSCLC progression, gain- and loss-of-function experiments were carried out. qRT-PCR analysis revealed that all the 4 NSCLC cell lines tested had significantly lower levels of LIFR-AS1 and
ZNF471 than BEAS-2B cells (Fig. 3a). Conversely, the levels of miR-942-5p were significantly increased in NSCLC cell lines compared to BEAS-2B cells. Ectopic expression of LIFR-AS1 inhibited cell migration and invasion in PC-9 cells (Fig. 3b, c), where there was a low level of endogenous LIFR-AS1. Conversely, silencing of LIFRAS1 caused a promotion of cell migration and invasion in A549 cells (Fig. 3d, e). We also explored the influence of LIFR-AS1 on NSCLC cell proliferation. As determined by MTT assays, overexpression or knockdown of LIFR-AS1 did not alter the proliferation capacity of NSCLC cells (Additional file 1: Figure S4).

To evaluate the effect of LIFR-AS1 on tumor metastasis in vivo, we injected the indicated PC-9 cells into nude mice through the tail vein. Analysis of metastatic activity by bioluminescence imaging revealed that LIFR-AS1overexpressing PC-9 cells showed decreased lung metastatic activity compared to control cells (Fig. 4a, b). Histological examination further demonstrated that LIFR-AS1 overexpression resulted in reduced numbers of metastatic lesions in the lung (Fig. 4c, d). Taken together, these data indicate that LIFR-AS1 suppresses NSCLC metastasis.

\section{LIFR-AS1 exerts anti-invasive activity in NSCLC by sponging miR-942-5 $p$ and inducing ZNF471}

Next, we asked whether the miR-942-5p/ZNF471 axis was involved in LIFR-AS1-mediated suppression of NSCLC cell invasion. To this end, we performed rescue experiments by overexpressing miR-942-5p or knocking down ZNF471 in LIFR-AS1-overexpressed PC-9 cells. We found that enforced expression of miR-942-5p significantly restored the migration and invasion capacity of LIFR-AS1-overexpressing cells (Fig. 5a, b). Similarly, depletion of ZNF471 (Fig. 5c) reversed the inhibitory effect of LIFR-AS1 on the migration (Fig. 5d) and invasion (Fig. 5e) of PC-9 cells. In addition, overexpression of ZNF471 inhibited the invasion of PC-9 cells (Fig. 5f). Collectively, these observations suggest that ZNF471 acts as a downstream effector of LIFR-AS1 in restraining NSCLC cell invasion.

\section{Clinical significance of LIFR-AS1 in NSCLC}

To investigate the clinical relevance of LIFR-AS1 expression in NSCLC, we measured the expression levels of LIFR-AS1 in 73 pairs of NSCLC and corresponding noncancerous tissues. We observed that LIFR-AS1 expression was downregulated in the NSCLC tissues compared with adjacent normal tissues $(P<0.0001$; Fig. 6a). The downregulation of LIFR-AS1 was significantly correlated with advanced TNM stage $(P=0.0321)$ and lymph node metastasis $(P=0.0047$; Table 1$)$. Kaplan-Meier analysis based on lung adenocarcinoma TCGA dataset revealed 

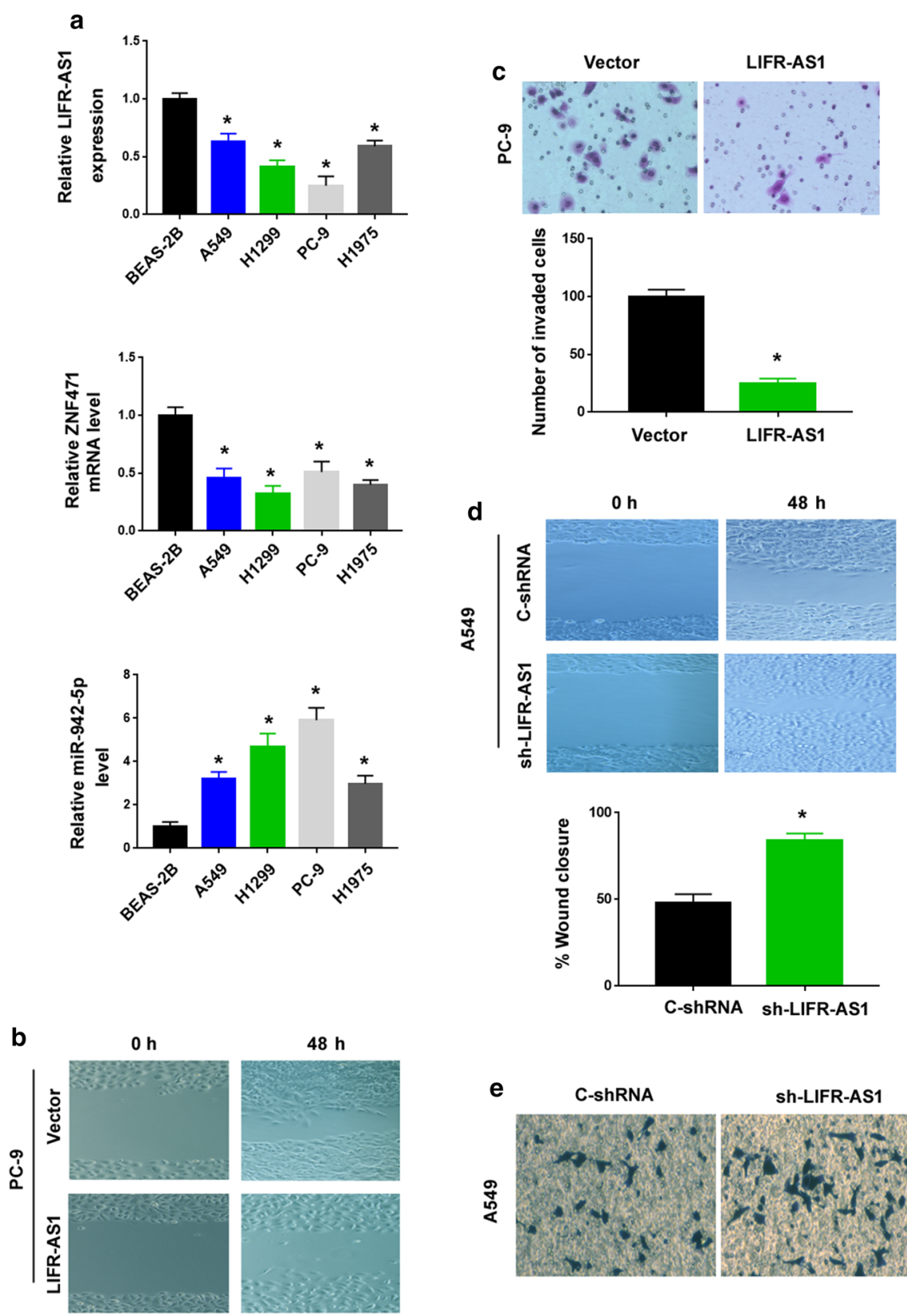

$48 \mathrm{~h}$
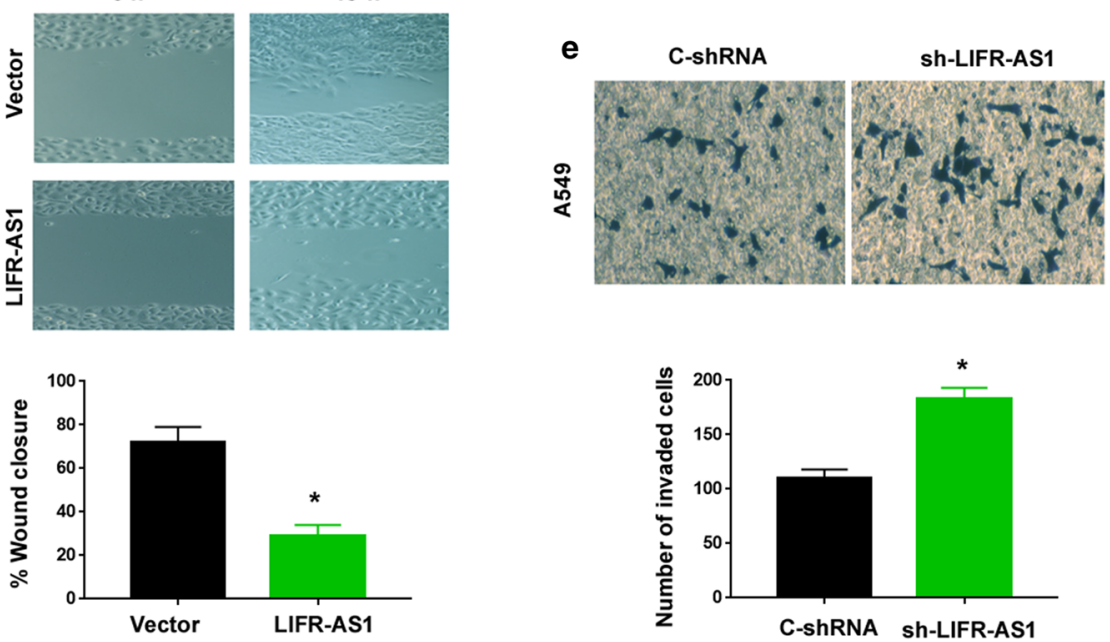

Fig. 3 LIFR-AS1 suppresses NSCLC cell migration and invasion. a Analysis of LIFR-AS1, ZNF471, and miR-942-5p levels in NSCLC cell lines and BEAS-2B cells. ${ }^{*} P<0.05$ vs. BEAS-2B cells. $\mathbf{b}$ Wound-healing assays showed that overexpression of LIFR-AS1 suppressed the migration of PC-9 cells. c Transwell invasion assays revealed that overexpression of LIFR-AS1 inhibited the invasion of PC-9 cells. $\mathbf{d}$ Knockdown of LIFR-AS1 promoted the migration of A549 cells. e Silencing of LIFR-AS1 enhanced the invasion of A549 cells. ${ }^{*} P<0.05$ 

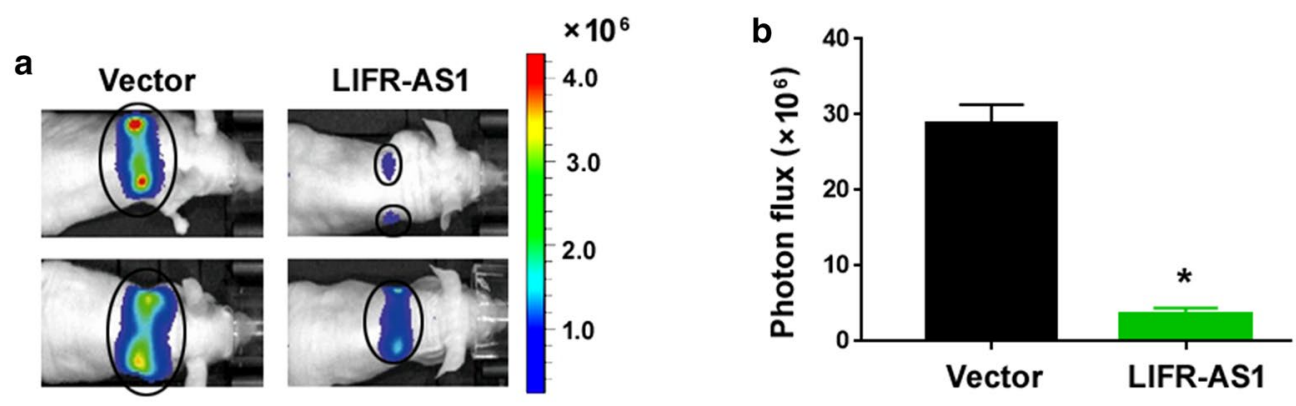

C

Vector

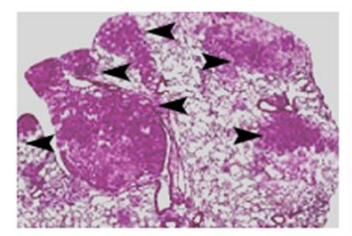

LIFR-AS1

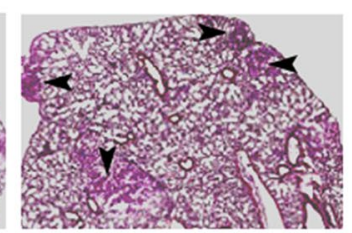

\section{d}

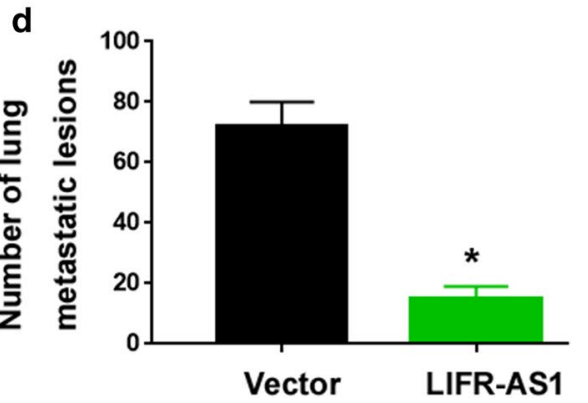

Fig. 4 LIFR-AS1 suppresses NSCLC metastasis in vivo. a, b LIFR-AS1-overexpressing and control PC-9 cells were injected to nude mice through the tail vein. Bioluminescence images were captured 6 weeks after cell injection. $\mathbf{c}$, $\mathbf{d}$ Histological examination showed LIFR-AS1 overexpression resulted in reduced numbers of metastatic lesions in the lung. Scale bar $=50 \mu \mathrm{m} .{ }^{*} P<0.05$ vs. the vector group

that low expression of LIFR-AS1 was significantly correlated with a reduced overall survival $(P=0.0099$; Fig. 6b). These data demonstrate that LIFR-AS1 expression is a favorable prognostic factor in NSCLC.

\section{Discussion}

In this work, we validate LIFR-AS1 as a key miR-942-5pinteracting lncRNA in NSCLC cells. LIFR-AS1 carries the binding site for miR-942-5p, and overexpression of miR-942-5p decreases the expression of LIFR-AS1 in NSCLC cells. Analysis of TCGA data reveals a negative correlation between miR-942-5p and LIFR-AS1 in lung adenocarcinoma (Additional file 1: Figure S5). Given that miR-942-5p has tumor-promoting effects on NSCLC [17], we speculated that LIFR-AS1 may play an important role in NSCLC progression. In line with this hypothesis, we show that LIFR-AS1 is downregulated in NSCLC tissues and cells, and ectopic expression of LIFR-AS1 leads to an inhibition of NSCLC cell migration and invasion. Conversely, depletion of LIFR-AS1 enhances the migration and invasion of NSCLC cells. These results point toward LIFR-AS1 as a tumor suppressor in NSCLC.

Dysregulation of lncRNAs is frequently detected in cancers, suggesting the potential as a biomarker for cancer progression [7-9]. Our data indicate that downregulation of LIFR-AS1 is significantly associated with advanced TNM stage and lymph node metastasis of
NSCLC patients. Moreover, LIFR-AS1 downregulation predicts poor prognosis in NSCLC. Therefore, LIFR-AS1 may serve as a novel prognostic marker for NSCLC. The clinical findings also suggest the therapeutic potential of LIFR-AS1 in preventing NSCLC progression.

It has been suggested that IncRNAs can function as ceRNA for miRNAs to modulate diverse biological processes [26, 27]. For example, Xu et al. [27] reported that the IncRNA SPRY4-IT1 promotes cell proliferation, migration, and invasion in cholangiocarcinoma by sponging miR-101-3p. Having identification of the interaction between LIFR-AS1 and miR-942-5p, we checked whether LIFR-AS1 exerts its tumor-suppressive effects through inhibition of miR-942-5p activity. Interestingly, we find that LIFR-AS1-mediated inhibition of NSCLC cell migration and invasion is rescued by ectopic expression of miR-942-5p. Biochemically, both LIFR-AS1 and miR-942-5p are enriched in Ago2 immunoprecipitates from NSCLC cells. These results suggest that sponging miR-942-5p accounts for the anti-invasive activity of LIFR-AS1 in NSCLC.

It has been documented that miR-942-5p can target BARX2 in NSCLC [17]. However, we did not detect the regulation of BARX2 by LIFR-AS1. This result suggest that LIFR-AS1 likely interferes with miR-942-5p binding to other target mRNAs. This hypothesis is supported by the finding that LIFR-AS1 can prevent 

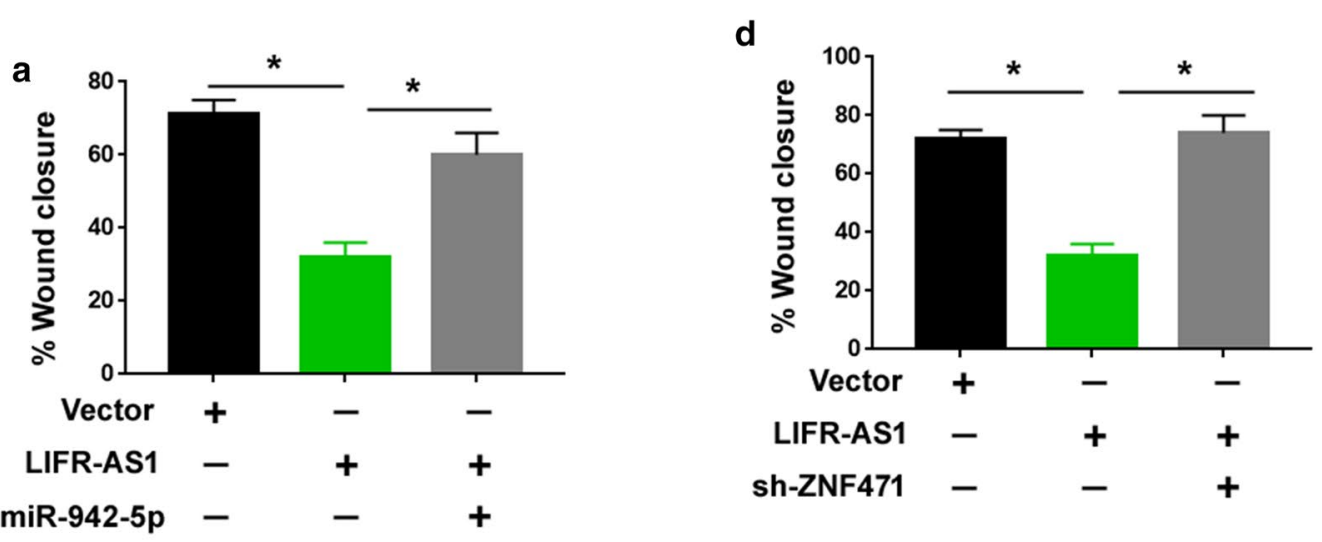

b
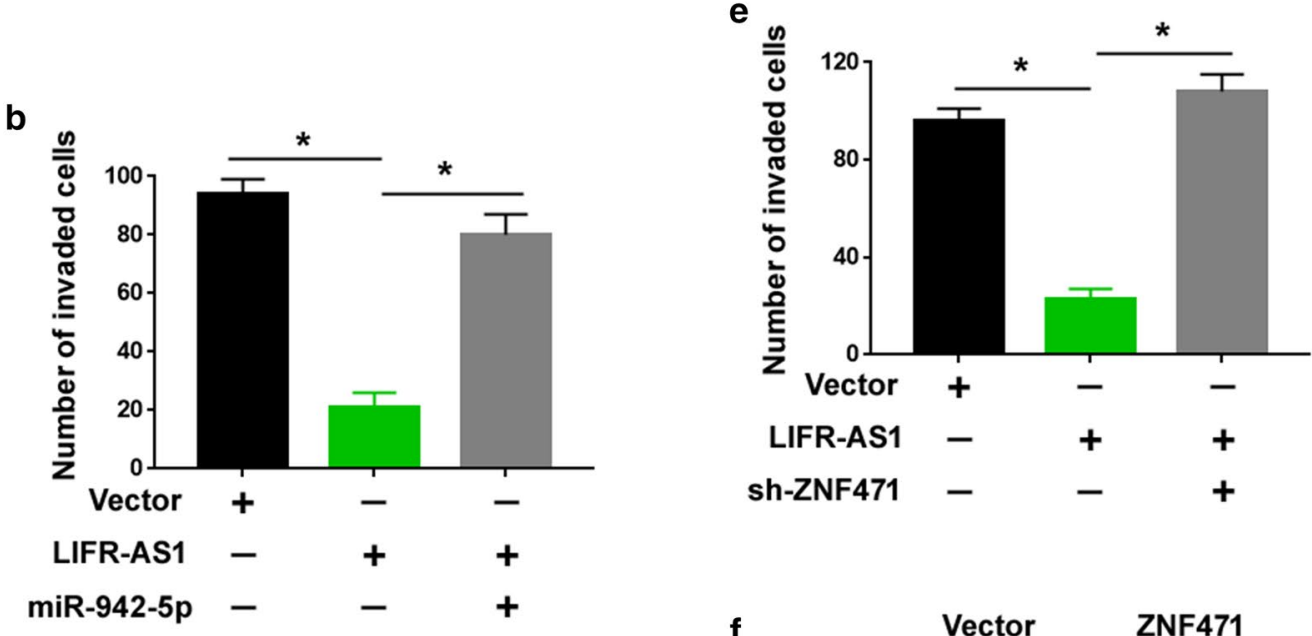

$\mathbf{f}$
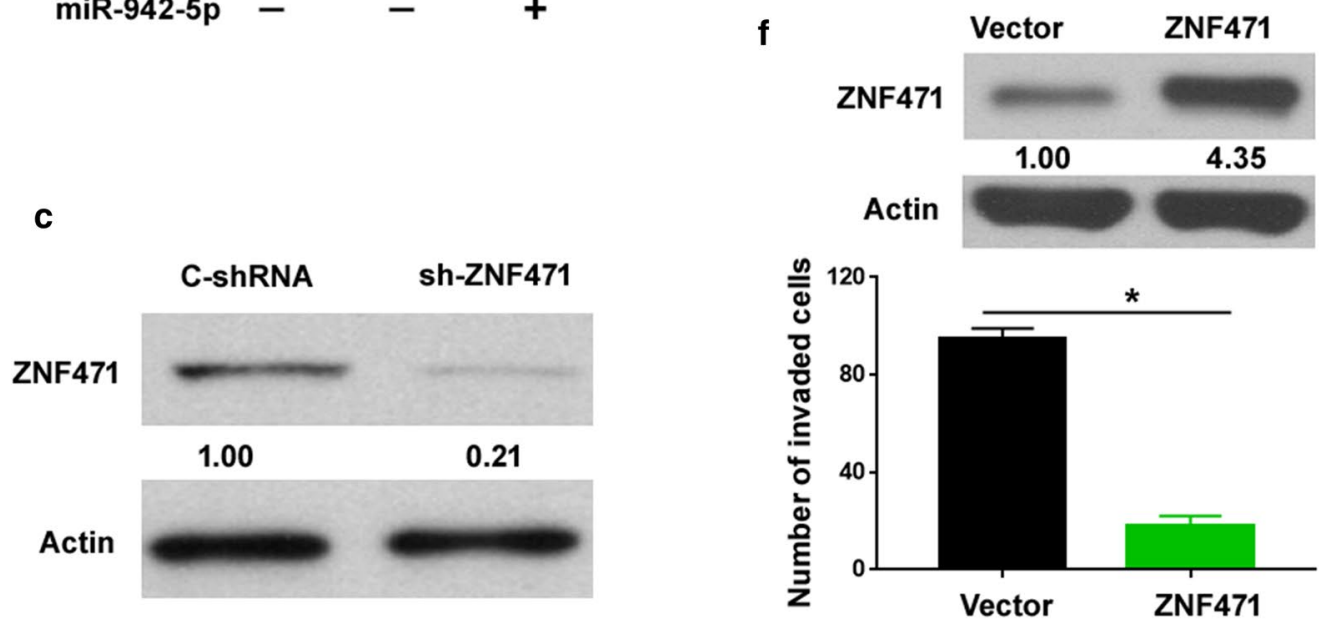

Fig. 5 LIFR-AS1 exerts anti-invasive activity in NSCLC by sponging miR-942-5p and inducing ZNF471. a, d PC-9 cells were transfected with indicated constructs and subjected to wound-healing assays. b, e PC-9 cells were transfected with indicated constructs and subjected to Transwell invasion assays. $\mathbf{c}$ Western blot analysis of ZNF471 protein levels in PC-9 cells transfected with control shRNA (C-shRNA) or ZNF471-targeting shRNA (sh-ZNF471). $\mathbf{f}$ Overexpression of ZNF471 inhibited the invasion of PC-9 cells. ${ }^{*} P<0.05$

the repression of ZNF471 by miR-942-5p. We provide first evidence that ZNF471 is a direct target gene of miR-942-5p. RIP assays reveal that miR-942-5p overexpression leads to an enrichment of ZNF471 mRNA in Ago2 immunoprecipitates. Enforced expression of miR-942-5p significantly decreases the expression of ZNF471 in NSCLC cells, whereas overexpression of LIFR-AS1 causes an upregulation of ZNF471. 


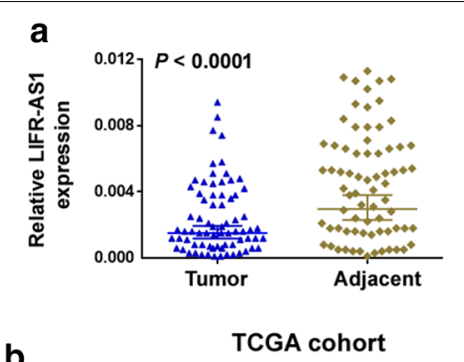

b

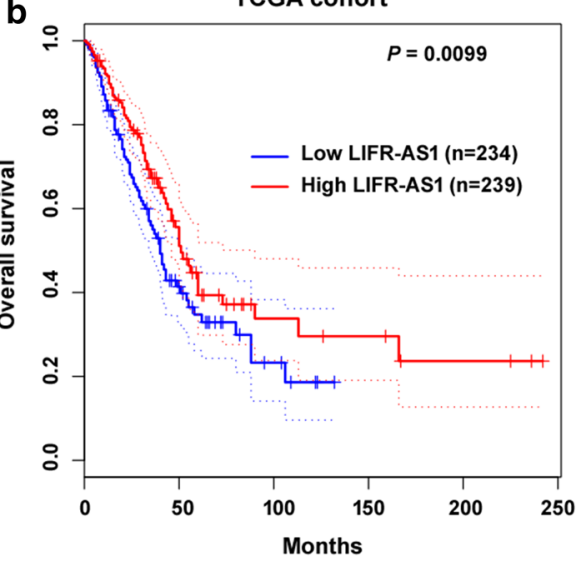

C

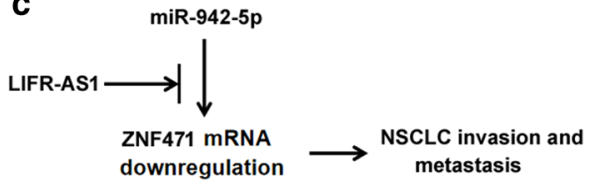

Fig. 6 Clinical significance of LIFR-AS1 in NSCLC. a Measurement of the expression levels of LIFR-AS1 in 73 pairs of NSCLC and corresponding noncancerous tissues. b Kaplan-Meier analysis based on lung adenocarcinoma TCGA dataset revealed that low expression of LIFR-AS1 was significantly correlated with a reduced overall survival. Statistical differences were determined by the log-rank test. c Schematic model showing that LIFR-AS1 sponges miR-942-5p to derepress ZNF471, consequently blocking NSCLC cell invasion and metastasis

In addition, LIFR-AS1 is positively correlated with ZNF471 mRNA in lung adenocarcinoma, according to TCGA datasets (Additional file 1: Figure S6). It has been reported that ZNF471 can block the proliferation and invasion of gastric cancer cells [5]. Consistently, we also validate ZNF471 as a tumor suppressor in NSCLC. We show that overexpression of ZNF471 restrains the invasion of PC-9 cells. Moreover, depletion of ZNF471 or overexpression of miR-942-5p restores the invasive property in LIFR-AS1-overexpressing NSCLC cells. Taken together, we propose a model in which LIFRAS1 sponges miR-942-5p to derepress ZNF471, consequently blocking NSCLC cell invasion and metastasis (Fig. 6c).

\section{Conclusion}

Our findings indicate that LIFR-AS1 acts as a sponge of miR-942-5p and prevents miR-942-5p-mediated repression of ZNF471, consequently impairing NSCLC cell invasion and metastasis. LIFR-AS1 downregulation is associated with poor prognosis of NSCLC patients. Therefore, we suggest LIFR-AS1 as a possible therapeutic target for NSCLC.

\section{Supplementary information}

Supplementary information accompanies this paper at https://doi. org/10.1186/s12935-020-01228-5.

Additional file 1: Table S1. Prediction of miR-942-5p-interacting IncRNAs with using the Encyclopedia of RNA Interactomes (ENCORI) program. Figure S1. Effect of LIFR-AS1 overexpression on the expression of miR-942-5p in NSCLC cells. n.s. indicates no significance. Figure S2. Effect of LIFR-AS1 overexpression on the expression of BARX2 in NSCLC cells. (A) Measurement of BARX2 mRNA levels. (B) Western blot analysis of BARX2 protein levels. n.s. indicates no significance. Figure S3. Effect of miR-942-5p overexpression on the abundance of RND3, KDM5A, and CCBE1 mRNAs in NSCLC cells. n.s. indicates no significance. Figure S4. Effect of LIFR-AS1 overexpression or knockdown on the proliferation of (A) PC-9 and (B) A549 cells. Cell proliferation was determined by direct cell counting at indicated time points after plating. n.s. indicates no significance. Figure S5. Analysis of TCGA data reveals a negative correlation between miR-942-5p and LIFR-AS1 in lung adenocarcinoma. Figure S6. Analysis of TCGA data reveals a positive correlation between LIFR-AS1 and ZNF471 mRNA in lung adenocarcinoma.

\section{Abbreviations}

ceRNA: Competitive endogenous RNA; IncRNA: Long non-coding RNA; NSCLC: Non-small cell lung cancer; qRT-PCR: Quantitative real-time PCR; shRNA: Short hairpin RNA; UTR: Untranslated region; ZNF471: Zinc-finger protein 471.

\section{Acknowledgements}

Not applicable.

\section{Authors' contributions}

$\mathrm{QW}, \mathrm{JW}, \mathrm{HH}$, and DSH were involved in the study conception and design and data analysis. YJ, YH, HYF, GZ, XCZ, YJW, and CJL performed experiments and participated in the data collection. QW drafted the manuscript. All authors accepted the final version. All authors read and approved the final manuscript.

\section{Funding}

This study was supported by the fourth batch of Wuhan Excellent Young and Middle-aged Medical backbone Researcher Program of China and the 2019 Hubei Province Postdoctoral Science and Technology Foundation of China.

\section{Availability of data and materials}

Not applicable

\section{Ethics approval and consent to participate}

The animal experiments were approved by the Institutional Animal Care and Use Committee of Hubei Cancer Hospital (Wuhan, China). Written informed consent was obtained from each patient. This study was approved by the Institutional Review Board of Hubei Cancer Hospital.

\section{Consent for publication}

Not applicable.

Competing interests

The authors declare that they have no competing interests. 


\begin{abstract}
Author details
${ }^{1}$ Department of Radiotherapy, Hubei Cancer Hospital, Tongji Medical College, Huazhong University of Science and Technology, 116 Zhuodaoquan South Road, Wuhan 430070, China. ${ }^{2}$ Department of Oncology, The Fifth Hospital of Wuhan, Wuhan, China. ${ }^{3}$ WuHan University, Wuhan, China. ${ }^{4}$ Department of Pneumology, The Fifth Hospital of Wuhan, Wuhan, China. ${ }^{5}$ Department of General Surgery, The Fifth Hospital of Wuhan, Wuhan, China.
\end{abstract}

Received: 12 January 2020 Accepted: 23 April 2020

Published online: 24 May 2020

\section{References}

1. Ettinger DS, Wood DE, Aggarwal C, Aisner DL, Akerley W, Bauman JR, et al. NCCN guidelines insights: non-small cell lung cancer, version 1.2020. J Natl Compr Canc Netw. 2019;17:1464-72.

2. Topalian SL, Hodi FS, Brahmer JR, Gettinger SN, Smith DC, McDermott DF, et al. Five-year survival and correlates among patients with advanced melanoma, renal cell carcinoma, or non-small cell lung cancer treated with nivolumab. JAMA Oncol. 2019. https://doi.org/10.1001/jamao ncol.2019.2187.

3. Manshouri R, Coyaud E, Kundu ST, Peng DH, Stratton SA, Alton K, et al. ZEB1/NuRD complex suppresses TBC1D2b to stimulate E-cadherin internalization and promote metastasis in lung cancer. Nat Commun. 2019;10:5125

4. Bhat S, Kabekkodu SP, Jayaprakash C, Radhakrishnan R, Ray S, Satyamoorthy K. Gene promoter-associated CpG island hypermethylation in squamous cell carcinoma of the tongue. Virchows Arch. 2017:470:445-54

5. Cao L, Wang S, Zhang Y, Wong KC, Nakatsu G, Wang X, et al. Zincfinger protein 471 suppresses gastric cancer through transcriptionally repressing downstream oncogenic PLS3 and TFAP2A. Oncogene. 2018:37:3601-16.

6. Sun R, Xiang T, Tang J, Peng W, Luo J, Li L, et al. 19q13 KRAB zinc-finger protein ZNF471 activates MAPK10/JNK3 signaling but is frequently silenced by promoter CpG methylation in esophageal cancer. Theranostics. 2020;10:2243-59.

7. You BH, Yoon JH, Kang H, Lee EK, Lee SK, Nam JW. HERES, a IncRNA that regulates canonical and noncanonical Wnt signaling pathways via interaction with EZH2. Proc Natl Acad Sci USA. 2019. https://doi.org/10.1073/ pnas.1912126116.

8. Yang R, Liu N, Chen L, Jiang Y, Shi Y, Mao C, et al. GIAT4RA functions as a tumor suppressor in non-small cell lung cancer by counteracting Uchl3mediated deubiquitination of LSH. Oncogene. 2019;38:7133-45.

9. Jia Y, Duan Y, Liu T, Wang X, Lv W, Wang M, et al. LncRNA TTN-AS1 promotes migration, invasion, and epithelial mesenchymal transition of lung adenocarcinoma via sponging miR-142-5p to regulate CDK5. Cell Death Dis. 2019;10:573.

10. Liu K, Yao H, Wen Y, Zhao H, Zhou N, Lei S, et al. Functional role of a long non-coding RNA LIFR-AS1/miR-29a/TNFAIP3 axis in colorectal cancer resistance to pohotodynamic therapy. Biochim Biophys Acta Mol Basis Dis. 2018;1864:2871-80

11. Xu F, Li H, Hu C. LIFR-AS1 modulates Sufu to inhibit cell proliferation and migration by miR-197-3p in breast cancer. Biosci Rep. 2019. https://doi. org/10.1042/BSR20180551.

12. Liao K, Lin Y, Gao W, Xiao Z, Medina R, Dmitriev P, et al. Blocking IncRNA MALAT1/miR-199a/ZHX1 axis inhibits glioblastoma proliferation and progression. Mol Ther Nucleic Acids. 2019;18:388-99.
13. Ge C, Wu S, Wang W, Liu Z, Zhang J, Wang Z, et al. miR-942 promotes cancer stem cell-like traits in esophageal squamous cell carcinoma through activation of Wnt/ $\beta$-catenin signalling pathway. Oncotarget. 2015;6:10964-77.

14. Shan Z, An N, Qin J, Yang J, Sun H, Yang W. Long non-coding RNA Linc00675 suppresses cell proliferation and metastasis in colorectal cancer via acting on miR-942 and Wnt/ $\beta$-catenin signaling. Biomed Pharmacother. 2018;101:769-76.

15. Zhang Q, Zhu B, Qian J, Wang K, Zhou J. miR-942 promotes proliferation and metastasis of hepatocellular carcinoma cells by inhibiting RRM2B. Onco Targets Ther. 2019;12:8367-78.

16. Zhang J, Zhang Z, Sun J, Ma Q, Zhao W, Chen X, et al. MiR-942 regulates the function of breast cancer cell by targeting FOXA2. Biosci Rep. 2019. https://doi.org/10.1042/bsr20192298.

17. Yang F, Shao C, Wei K, Jing X, Qin Z, Shi Y, et al. miR-942 promotes tumor migration, invasion, and angiogenesis by regulating EMT via BARX2 in non-small-cell lung cancer. J Cell Physiol. 2019;234:23596-607.

18. Huang MJ, Zhao JY, Xu JJ, Li J, Zhuang YF, Zhang XL. IncRNA ADAMTS9AS2 controls human mesenchymal stem cell chondrogenic differentiation and functions as a ceRNA. Mol Ther Nucleic Acids. 2019:18:533-45.

19. Livak KJ, Schmittgen TD. Analysis of relative gene expression data using real-time quantitative PCR and the 2(-Delta Delta C(T)) Method. Methods. 2001;25:402-8

20. Lu S, Sun Z, Tang L, Chen L. LINC00355 promotes tumor progression in HNSCC by hindering MicroRNA-195-mediated suppression of HOXA10 expression. Mol Ther Nucleic Acids. 2019;19:61-71.

21. Chen Y, Zhao H, Li H, Feng X, Tang H, Zhang J, et al. LINC01234/MicroRNA 31-5p/MAGEA3 axis mediates the proliferation and chemoresistance of hepatocellular carcinoma cells. Mol Ther Nucleic Acids. 2019:19:168-78.

22. Zhang P, Tang WM, Zhang H, Li YQ, Peng Y, Wang J, et al. MiR-646 inhibited cell proliferation and EMT-induced metastasis by targeting FOXK1 in gastric cancer. Br J Cancer. 2017;117:525-34.

23. Zhu Z, Rong Z, Luo Z, Yu Z, Zhang J, Qiu Z, et al. Circular RNA circNHSL1 promotes gastric cancer progression through the miR-1306-3p/SIX1/ vimentin axis. Mol Cancer. 2019;18:126.

24. Gao P, Wang H, Yu J, Zhang J, Yang Z, Liu M, et al. miR-3607-3p suppresses non-small cell lung cancer (NSCLC) by targeting TGFBR1 and CCNE2. PLoS Genet. 2018:14:e1007790.

25. Gebert LFR, MacRae IJ. Regulation of microRNA function in animals. Nat Rev Mol Cell Biol. 2019:20:21-37.

26. He Z, You C, Zhao D. Long non-coding RNA UCA1/miR-182/PFKFB2 axis modulates glioblastoma-associated stromal cells-mediated glycolysis and invasion of glioma cells. Biochem Biophys Res Commun. 2018;500:569-76.

27. Xu Y, Yao Y, Jiang X, Zhong X, Wang Z, Li C, et al. SP1-induced upregulation of IncRNA SPRY4-IT1 exerts oncogenic properties by scaffolding EZH2/LSD1/DNMT1 and sponging miR-101-3p in cholangiocarcinoma. J Exp Clin Cancer Res. 2018;37:81

\section{Publisher's Note}

Springer Nature remains neutral with regard to jurisdictional claims in published maps and institutional affiliations.

Ready to submit your research? Choose BMC and benefit from

- fast, convenient online submission

- thorough peer review by experienced researchers in your field

- rapid publication on acceptance

- support for research data, including large and complex data types

- gold Open Access which fosters wider collaboration and increased citations

- maximum visibility for your research: over 100M website views per year

At BMC, research is always in progress.

Learn more biomedcentral.com/submissions 